OPEN ACCESS

Edited by:

Maoteng Li,

Huazhong University of Science and Technology, China

Reviewed by:

Chunhua Fu,

Huazhong University of Science and Technology, China

Yan Long,

Chinese Academy of Agricultural

Sciences, China

Jia Liu,

Chinese Academy of Agricultural

Sciences, China

${ }^{*}$ Correspondence:

Jianjun Chen

jjchen@ufl.edu

Chunying Zhang

mayzhang55@163.com

Dongming Pan

pdm666@126.com

Specialty section:

This article was submitted to Crop Science and Horticulture,

a section of the journal

Frontiers in Plant Science

Received: 20 July 2016 Accepted: 10 October 2016 Published: 25 October 2016

Citation:

Wei X, Chen J, Zhang C and Pan D (2016) Differential Gene Expression in Rhododendron fortunei Roots

Colonized by an Ericoid Mycorrhizal Fungus and Increased Nitrogen Absorption and Plant Growth

Front. Plant Sci. 7:1594. doi: 10.3389/fpls.2016.01594

\section{Differential Gene Expression in} Rhododendron fortunei Roots Colonized by an Ericoid Mycorrhizal Fungus and Increased Nitrogen Absorption and Plant Growth

\author{
Xiangying Wei ${ }^{1,2}$, Jianjun Chen ${ }^{1,2 *}$, Chunying Zhang ${ }^{3 *}$ and Dongming Pan ${ }^{1 *}$ \\ ${ }^{1}$ College of Horticulture, Fujian Agriculture and Forestry University, Fuzhou, China, ${ }^{2}$ Department of Environmental \\ Horticulture and Mid-Florida Research and Education Center, Institute of Food and Agricultural Sciences, University of \\ Florida, Apopka, FL, USA, ${ }^{3}$ Shanghai Academy of Landscape Architecture Science and Planning, Shanghai, China
}

Ericoid mycorrhizal (ERM) fungi are specifically symbiotic with plants in the family Ericaceae. Little is known thus far about their symbiotic establishment and subsequent nitrogen $(\mathrm{N})$ uptake at the molecular level. The present study devised a system for establishing a symbiotic relationship between Rhododendron fortunei Lindl. and an ERM fungus (Oidiodendron maius var. maius strain Om19), quantified seedling growth and $\mathrm{N}$ uptake, and compared transcriptome profiling between colonized and uncolonized roots using RNA-Seq. The Om19 colonization induced 16,892 genes that were differentially expressed in plant roots, of which 14,364 were upregulated and 2,528 were downregulated. These genes included those homologous to ATP-binding cassette transporters, calcium/calmodulin-dependent kinases, and symbiosis receptor-like kinases. $\mathrm{N}$ metabolism was particularly active in Om19-colonized roots, and 51 genes were upregulated, such as nitrate transporters, nitrate reductase, nitrite reductase, ammonium transporters, glutamine synthetase, and glutamate synthase. Transcriptome analysis also identified a series of genes involving endocytosis, Fc-gamma R-mediated phagocytosis, glycerophospholipid metabolism, and Gonadotropin-releasing hormone $(\mathrm{GnRH})$ signal pathway that have not been reported previously. Their roles in the symbiosis require further investigation. The Om19 colonization significantly increased $\mathrm{N}$ uptake and seedling growth. Total $\mathrm{N}$ content and dry weight of colonized seedlings were 36.6 and $46.6 \%$ greater than control seedlings. This is the first transcriptome analysis of a species from the family Ericaceae colonized by an ERM fungus. The findings from this study will shed light on the mechanisms underlying symbiotic relationships of ericaceous species with ERM fungi and the symbiosis-resultant $\mathrm{N}$ uptake and plant growth.

Keywords: Ericaceae, ericoid mycorrhiza, nitrate uptake, Rhododendron fortunei, symbiosis, transcriptome analysis 


\section{INTRODUCTION}

Fungi play vital roles in many microbiological and ecological processes. The mutualistic association of plant roots with mycorrhizal fungi is the most widespread terrestrial symbiosis (Parniske, 2008). Host plants provide fungi with carbon compounds for growth and reproduction, while the fungi enhance host nutrient uptake and water absorption and improve disease resistance (Harrison, 2005). There are two types of mycorrhiza: ectomycorrhizas and endomycorrhizas, depending on whether the fungal hyphae colonize the root intercellular spaces or develop inside cells. Endomycorrhizas are further divided into ericoid, orchid, and arbuscular mycorrhizas (AM; Bonfante and Genre, 2010).

Endomycorrhizal colonization is a complex process, and current research has largely focused on AM fungi (Stracke et al., 2002; Akiyama et al., 2005). At the presymbiotic stage of colonization, plant roots produce and release carotenoid phytohormones, specifically strigolactones. Two GRAS-type transcription factors [named after the first three members: GIBBERELLIC-ACID INSENSITIVE (GAI), REPRESSOR of GAI (RGA) and SCARECROW (SCR)], NSP1 and NSP2 (nodulation-signaling pathway 1 and 2), which are both indispensable for nodulation in legumes (Kaló et al., 2005; Smit et al., 2005), have been implicated in the regulation of strigolactone biosynthesis. Strigolactones induce spore germination and hyphal growth and branching (Gutjahr and Parniske, 2013). Fungi produce mycorrhizal factors to induce calcium oscillations in root epidermal cells to activate plant symbiosis-related genes. Some of these identified genes include DMI1 (does not make infections), DMI2, and DMI3. DMI1 encodes a predicted ion channel (Ané et al., 2004) which acts upstream of calcium spiking. DMI2 encodes a symbiosis receptor-kinase (SymRK) or nodulation receptor kinase (NORK) that functions in the nod-factor perception/transduction system to initiate a signal cascade leading to nodulation (Endre et al., 2002). DMI3 is a calcium/calmodulin-dependent kinase (CCaMK; Lévy et al., 2004; Mitra et al., 2004). These genes are important for both AM formation and root nodulation. AM fungi form special types of appressoria called hyphopodia. As a consequence of sequential chemical and mechanical stimulation, plant cells produce a pre-penetration apparatus (PPA). A fungal hypha that extends from the hyphopodium enters the PPA, which guides the fungus through root cells toward the cortex. At present, the signals triggering the formation of the PPA are unknown. Phenotypic analysis of Medicago truncatula symbiotic mutants shows that SymRK/DMI2 are required for PPA induction and that DMI3 is required for a subset of genes to be induced during PPA formation. A potential plant receptor for fungal chitin derivatives has been identified in Parasponia andersonii (Op den Camp et al., 2011). Silencing of this putative receptor, a LysM receptor kinase, which is closely related to the NFR5/NFP Nod factor receptor kinases from the legumes Lotus and Medicago, led to loss of nodulation and AM formation (Op den Camp et al., 2011).

Mycorrhizal symbiosis can significantly enhance plant roots in nutrient acquisition. Mycorrhiza-mediated phosphate uptake has been well documented (Karandashov and Bucher, 2005). Recent studies show that mycorrhizal colonization can also improve plant roots in $\mathrm{N}$ absorption (Govindarajulu et al., 2005; Tian et al., 2010). AM fungi access $\mathrm{N}$ and transport absorbed $\mathrm{N}$ via arginine from extra- to intra-radical mycelia; and the arginine is broken down through the urease cycle into ammonium for transport into the plant (Govindarajulu et al., 2005; Tian et al., 2010). Ammonium transporters (AMTs) have been identified to be responsible for uptake of ammonium at the periarbuscular membrane (Parniske, 2008). Plants also possess another set of transporters for uptake of nitrate $\left(\mathrm{NO}_{3}{ }^{-}\right): \mathrm{NO}_{3}{ }^{-}$ transporters (NRTs), which are encoded by two distinct gene families (NRT1 and NRT2). The NRT1 family mainly regulates the low-affinity transport system (LATS), and the NRT2 family regulates high-affinity transport system (HATS; von Wittgenstein et al., 2014). Thus, roots symbiotically associated AM fungi have both mycorrhiza-mediated uptake and plant uptake pathways for acquisition of N (Bucking and Kafle, 2015).

Ericoid mycorrhizal (ERM) fungi are a distinctive type of endomycorrhiza, which forms symbiotic relationships specifically with roots of plants in the family Ericaceae, commonly known as the heath or heather family. The symbiosis allows host plants to adapt to a broad range of habitats, particularly acidic and infertile growing conditions (Cairney and Meharg, 2003). Until now, there is little information regarding the molecular basis of symbiosis between ERM fungi and plants from Ericaceae. Additionally, mechanisms regarding $\mathrm{N}$ uptake in plants of Ericaceae remain controversial. Cranberry (Vaccinium macrocarpon Ait.), a member of the family of Ericaceae, was reported to be unable to use $\mathrm{NO}_{3}{ }^{-}$as a sole source of $\mathrm{N}$ in hydroponic culture (Rosen et al., 1990; Smith, 1993). This has been explained by a notion that nitrification was typically assumed to be negligible at soil pH below 5.5 (Paul and Clark, 1989), a characteristic of the sphagnum bog habitat native to cranberry and many other Ericaceae (Read and Perez-Moreno, 2003; Read et al., 2004). The adaptation to such soil conditions has resulted in the loss of the capacity to absorb $\mathrm{NO}_{3}{ }^{-}$. A recent report, however, showed that inoculation with the fungus Rhizoscyphus ericae increased the capacity of cranberry to absorb $\mathrm{NO}_{3}^{-}$(Kosola et al., 2007), and the authors believe that $\mathrm{NO}_{3}{ }^{-}$may play a greater role in $\mathrm{N}$ nutrition of cranberry than previously thought and that high capacity for $\mathrm{NO}_{3}{ }^{-}$utilization is the ancestral state for the Ericaceae. Yin et al. (2010) also found that ERM fungi significantly increased the ability of $R$. fortunei to absorb $\mathrm{N}$, especially in the form of nitrate. Therefore, a better understanding of how ERM symbiosis enhances $\mathrm{N}$ uptake is not only biologically but also practically important as it could help improve production of some economically important horticultural crops, such as cranberry, blueberry, and rhododendron.

RNA-sequencing (RNA-Seq) has revolutionarily advanced sequence-based gene discovery (Wang et al., 2009). It provides a unique combination of transcriptome-wide coverage, sensitivity, and accuracy for a comprehensive view of gene expression changes for a specific developmental stage or physiological condition (Martin et al., 2013). RNA-Seq is rapidly becoming the method of choice for uncovering multiple facets of transcriptome 
to facilitate the biological applications including the investigation of plant-microbe interactions (Knief, 2014).

The present study was intended to explore the symbiotic relationships of ericaceous plants with ERM fungi and mechanisms underlying $\mathrm{N}$ acquisition using Rhododendron fortunei and Oidiodendron maius var. Maius strain Om19 as model organisms. A system to initiate their symbiosis was established, and subsequent $\mathrm{N}$ uptake and plant growth were examined. Using RNA-Seq, expression profiling of $R$. fortunei roots colonized by Om19 was analyzed against non-colonized control. A total of 16,892 differential-expressed genes (DEGs) were identified. Results from this study could shed light on molecular mechanisms governing the symbiotic establishment between ERM fungi and plants in the family Ericaceae and manifest how the symbiosis improves plant growth and $\mathrm{N}$ uptake.

\section{MATERIALS AND METHODS}

\section{Plant Material and Ericoid Mycorrhizal Fungus}

Seeds of $R$. fortunei were rinsed in running tap water for $2 \mathrm{~h}$ and surface sterilized four times, $5 \mathrm{~min}$ each, using $25 \%$ (v $\left.\mathrm{v}^{-1}\right)$ solution of commercial bleach $(8.25 \% \mathrm{NaOCl})$ followed by rinsing in sterile distilled water three times. The sterilized seeds were germinated on a half-strength Economou and Read medium (Economou and Read, 1984) supplemented with 1.5\% $\left(\mathrm{w} \mathrm{v}^{-1}\right)$ sucrose and $0.7 \%\left(\mathrm{w} \mathrm{v}^{-1}\right)$ agar with a $\mathrm{pH}$ of 5.2. The germination took place in a culture room under a 16$\mathrm{h}$ photoperiod provided by cool-white fluorescent lamps at a photon flux density of $50 \mathrm{mmol} \mathrm{m} \mathrm{m}^{-2} \mathrm{~s}^{-1}$. Meanwhile, a peatbased substrate was formulated by mixing dry Klasmann peat (Geeste, Germany) with dry sand (washed with deionized water and dried) at 2 to 1 ratio based on volume. A modified MelinNorkans (MMN) nutrient solution (Xiao and Berch, 1992) devoid of malt extract and glucose was prepared where the $\mathrm{N}$ source was replaced by $\mathrm{Ca}\left(\mathrm{NO}_{3}\right)_{2}$ to a final $\mathrm{N}$ concentration of $3.79 \mathrm{mM}$ and $\mathrm{pH}$ was adjusted to 5.2. The peat-based substrate was moistened with the modified MMN solution at 3 to 2 ratio by volume, and its $\mathrm{pH}$ was tested to be to 5.2. The substrate was filled into 120 cylindrical vessels $(400 \mathrm{~mL})$ with $100 \mathrm{~mL}$ each, covered with caps, and autoclaved at $121^{\circ} \mathrm{C}$ for $30 \mathrm{~min}$. Two months after seed germination, seedlings were transferred to 120 culture vessels containing the sterilized substrate, five seedlings per vessel.

Oidiodendron maius Om19 (Wei et al., 2016) was cultured on MMN agar medium (Molina and Palmer, 1982; Zhang et al., 2009). This strain was isolated from hair roots of $R$. fortunei grown in Huading Forest Park, Zhejiang Province, China. The sequence of this strain's rDNA internal transcribed space was submitted to the NCBI database under the accession number KU382495. After 2 weeks of culture on MMN, mycelia of Om19 were collected using a sterile 5-mm diameter cork borer. After removing extra medium to a thickness of $1 \mathrm{~mm}$, the $5-\mathrm{mm}$ diameter disks were cut into half (a surface area of $9.8 \mathrm{~mm}^{2}$ ) were inoculated into the peat-based substrate next to each of the five $R$. fortunei seedlings. A total of 60 vessels were inoculated, while the remaining 60 vessels without inoculation were considered the control treatment. The inoculated and uninoculated treatments were designated as JZ and WJZ, respectively. To record plant growth, seedlings from randomly selected six vessels per treatment were collected weekly for 10 weeks. Roots were washed away of the substrate debris, the fresh weight of five seedlings in each vessel were recorded after blotted with paper towel. Means were calculated, and data were presented as mean \pm SE.

\section{Microscopic Observation}

After fresh weight recoding, roots were immediately fixed in formaldehyde-acetic acid-ethanol (FAA) for $24 \mathrm{~h}$ and heated at $90^{\circ} \mathrm{C}$ for $1 \mathrm{~h}$ in $10 \% \mathrm{KOH}$. The roots were rinsed in water, acidified with $1 \% \mathrm{HCl}$, and stained in a lactophenol-trypan blue $\left(0.05 \%\right.$ trypan blue in lactophenol) for $5 \mathrm{~min}$ at $90^{\circ} \mathrm{C}$ (Phillips and Hayman, 1970; Zhang et al., 2008). Stained roots were cleared with fresh lactophenol. The roots were examined under light microscopy for the presence of fungus inside root cells. Root colonization was quantitatively assessed using the method described by Biermann and Linderman (1981) as the percentage of root length with internal hyphal coils. Root samples collected on week 6 were also observed under scanning electronic microscope (SEM) using the method described by BonfanteFasolo and Gianinazzi-Pearson (1979). All specimens were coated with gold and platinum and examined using a SEM at $20 \mathrm{kV}$.

\section{Plant Growth and N Uptake}

Based on the Om19 colonization results, a second experiment was carried out for determining biomass accumulation and tissue $\mathrm{N}$ content. The experiment was conducted in the same way as described above where 60 vessels were inoculated with Om19 as JZ treatment and the remaining 60 without inoculation as WJZ treatment. The experiment was arranged as a randomized complete block design with six blocks, and each treatment had 10 vessels per block. Plants were grown in the same conditions mentioned above without supplying any additional nutrient elements. After 6 weeks of inoculation, entire seedlings (shoots and roots) from both JZ and WJZ were harvested by carefully removing substrate from roots with running tap water. Seedlings from each block (50 seedlings) as a replicate were oven-dried at $80^{\circ} \mathrm{C}$ for at least $48 \mathrm{~h}$, dry mass (50 seedlings) was measured. Tissues were analyzed for total $\mathrm{N}$ using CNS Auto-Analyzer (VarioMAX, Elementar Americas, Mt. Laurel, NJ, USA).

\section{RNA Extraction and RNA-Seq}

A third experiment was carried out to prepare root materials for RNA extraction. The experiment was the same as the second experiment except the total number of culture vessels that were 300. Culture of Om19 was inoculated to 150 vessels (JZ), and the remaining 150 were control treatment without inoculation (WJZ). After 6 weeks of inoculation, roots were harvested by carefully removing substrate with running tap water and rinsed with sterile deionized water. After removing water with filter paper, roots harvested from JZ and WJZ treatments were, respectively, frozen in liquid $\mathrm{N}$ and stored at $-80^{\circ} \mathrm{C}$. Total RNA was extracted with TRIzol reagent (Invitrogen, USA) and treated with RNase-free DNase I (Takara Biotechnology, China). RNA 
integrity was assessed using the 2100 Bioanalyzer (Agilent). RNA integrity number (RIN) values were greater than 8 for all samples. Magnetic beads coated with oligo (dT) were used to isolate Poly (A)-containing mRNA. After synthesis of the first-strand cDNA using reverse transcriptase and random hexamer primers, the second strand of cDNA was synthesized using DNA polymerase I and RNaseH. Double-stranded cDNAs were subjected to endrepair using $\mathrm{T}_{4}$ DNA polymerase, Klenow fragment, and $\mathrm{T}_{4}$ polynucleotide kinase. The cDNAs were ligated with an adapter or an index adapter using $\mathrm{T}_{4}$ quick DNA ligase. The suitable adaptor-ligated fragments were selected for PCR amplification as templates. PCR was performed to enrich and amplify the selected fragments. The Agilent 2100 Bioanalyzer and ABI StepOnePlus Real-Time PCR System were used to quantify and assess the quality of the sample library. The cDNA library products were sequenced on the Illumina HiSeq ${ }^{\mathrm{TM}} 2000$ platform [The Beijing Genomics Institute (BGI), Shenzhen, China]. The transcriptome datasets are available at the NCBI Sequence Read Archive (SRA), under accession number SRP064996.

\section{Data Processing and De novo Assembly}

Raw data or reads generated by Illumina platform were cleaned by removing adapter sequences, empty reads, and low-quality sequences (reads with ambiguous bases ' $N$ '). The clean reads were compared against the genome of ERM fungi (Oidiodendron maius $^{1}$ ) using SOAP2 (Li et al., 2009). Any overlapping reads were discarded prior to performing assembly to ensure that all reads in the dataset were plant origin. Transcriptome de novo assembly was subsequently carried out using the short reads assembling program-Trinity (Grabherr et al., 2011), i.e., reads with overlaps were assembled to generate contigs, which were joined into scaffolds that were further assembled through gap filling to generate sequences called unigenes. Assembled unigenes from JZ and WJZ were taken into further process of sequence splicing, and redundancy was removed with sequence-clustering software to acquire non-redundant unigenes.

\section{Transcriptome Annotation}

Unigenes were aligned to several protein databases using BLASTx $\left(E\right.$-value $\left.<10^{-5}\right)$, including the NCBI non-redundant protein (Nr), Swiss-Prot (European Protein) database, the Kyoto Encyclopedia of Genes and Genomes (KEGG) pathway (Kanehisa et al., 2008), and the Cluster of Orthologous Groups of proteins $(\mathrm{COG})^{2}$ databases. Sequence directionality was assigned according to the best alignments. When the different databases gave different results, the following priority structure was used to choose one unigene: NCBI Nr, Swiss-Prot, KEGG, and COG. When a unigene failed to align to any of the four databases, ESTScan (Iseli et al., 1999) was used to predict its coding regions and ascertain its sequence direction.

\section{Differentially Expressed Unigenes}

To determine transcript abundance levels of unigenes, the uniquely mapped reads for a specific transcript were counted

\footnotetext{
${ }^{1}$ http://genome.jgi-psf.org/Oidmal/Oidma1.home.html
}

${ }^{2}$ http://www.ncbi.nlm.nih.gov/COG/ by mapping reads to assembled sequences using SOAP2 (Li et al., 2009). The RPKM (reads per Kb per million reads) values were calculated using Cufflinks program (Mortazavi et al., 2008; Trapnell et al., 2010). DEGs between the JZ and WJZ samples were obtained from RPKM values using a method modified by Audic and Claverie (1997). Fold changes for each unigene were calculated as the ratio of RPKM values. If the value of either JZ-RPKM or WJZ-RPKM was zero, 0.001 was used instead of 0 to measure the fold change. The significance of differential transcript abundance was computed using the FDR (False Discovery Rate) control method (Benjamini and Yekutieli, $2001)$ to justify the $p$-value, and only unigenes with an absolute fold change $\geq 2$ and a FDR significance score $\leq 0.001$ were used for subsequent steps of the analysis.

\section{Gene Ontology Enrichment and DEGs Pathway Analyses}

Gene ontology (GO) term annotation (molecular function, biological process, and cellular component) was analyzed using the Blast2go software (version 3.0) (Ashburner et al., 2000; Conesa et al., 2005) based on $\mathrm{Nr}$ database. After obtaining GO annotation for each unigene, WEGO software (Ye et al., 2006) was used to perform GO functional classification for all unigenes and to understand the distribution of gene functions at the macro level. The analysis first maps all DEGs to GO terms in the database by virtue of calculating gene numbers for every term, followed by an ultra-geometric test to find significantly enriched GO terms in DEGs compared to the transcriptome background. Calculated $p$-values were subjected to a Bonferroni Correction with a corrected $q$-value $\leq 0.05$ as a threshold. GO terms fulfilling this condition were defined as significantly enriched DEGs. This analysis recognized the main biological functions of identified DEGs. GO functional enrichment analysis also integrated the clustering analysis of expression patterns of DEGs. Additionally, the Blastall program was used to annotate the pathways of DEGs against the KEGG database (Kanehisa et al., 2008).

\section{qRT-PCR Analysis of Selected DEGs}

In order to verify some differential expressed genes, a fourth plant growth experiment was performed. Seedlings of $R$. fortunei were grown in 480 culture vessels of which 240 were inoculated with Om19 (JZ) and the remaining were uninoculated as control (WJZ). The experiment was a randomized complete block design with three blocks, and each treatment had 80 vessels per block. Twenty vessels per treatment were randomly selected from each block on weeks 1, 3, 6, and 8. Roots of JZ and WJZ from each block were, respectively, collected, and total RNAs were extracted using TRIzol reagent (Invitrogen, USA). qRT-PCR was carried out to analyze expression levels of 11 DEGs, which are homologous to SymRK, NORK, CCaMK, DM1, NRT1-1 and NRT1-2, AMT3, GS-1 and GS-2 (glutamine synthetase), GOGAT1 and GOGAT-2 (glutamate synthase). The sequence identity of the 11 DEGs to other plant species ranged from 69 to $86 \%$ (Supplementary Table S1). Gene specific primers were designed according to the cDNAs with Primer Premier software (version 5.0) (Supplementary Table S2). EF1 $\alpha$, previously tested as the 
most stably expressed gene, was used as an internal control. The first strand cDNA was synthesized using the PrimeScript II the first strand cDNA Synthesis Kit (Takara, Dalian, China). qRTPCR was performed in a $20 \mu \mathrm{L}$ reaction mixture containing 2x SYBR Master Premix Ex Taq II $12.5 \mu \mathrm{L}$ (Takara, Dalian, China), $1 \mu \mathrm{L}$ of cDNA template (1:5 dilution), and $1 \mu \mathrm{L}$ of each corresponding primer for the gene of interest and EF1 $\alpha$. qRTPCR of three biological replicates (from three blocks) for each treatment at four sampling periods was performed for $5 \mathrm{~s}$ at $95^{\circ} \mathrm{C}$, $10 \mathrm{~s}$ at $56^{\circ} \mathrm{C}$, and $20 \mathrm{~s}$ at $72^{\circ} \mathrm{C}$ using a LightCycler 480 II System. The relative expression levels were normalized and calibrated according to the $2^{-\Delta \Delta C T}$ method (Schmittgen and Livak, 2008). For a given gene, the relative expression level was expressed as mean \pm SE of three replicates.

\section{RESULTS}

\section{Seedling Growth and Om19 Colonization}

Seedlings grew healthily in the established system. Fresh weights of both WJZ and JZ seedlings continuously increased during the 10-week growth period (Figure 1). Starting from week 3, fresh weight of JZ seedlings increased significantly greater than those of WJZ. The significant growth increase corresponded to Om19 colonization of seedlings. The colonization rate in week 3 was $8 \%$ and increased to $70 \%$ in week 10 (Figure 1). Om19-colonized seedlings at the end of 10-week growth period had a mean root number of 8.2 and root length of $25.5 \mathrm{~mm}$ compared to 4.7 and $13.6 \mathrm{~mm}$ of the control seedlings. The mean leaf number and shoot height of Om19-colonized seedlings were 12.4 and $23.9 \mathrm{~mm}$ compared to 11.2 and $12.8 \mathrm{~mm}$ of control seedlings.

Light microscopy observation showed that mycelium was present on root surfaces of JZ seedlings as early as week 1 . Fungal entry points on the epidermal cells were observed in week 2; the intracellular hyphal growth was observed in epidermal

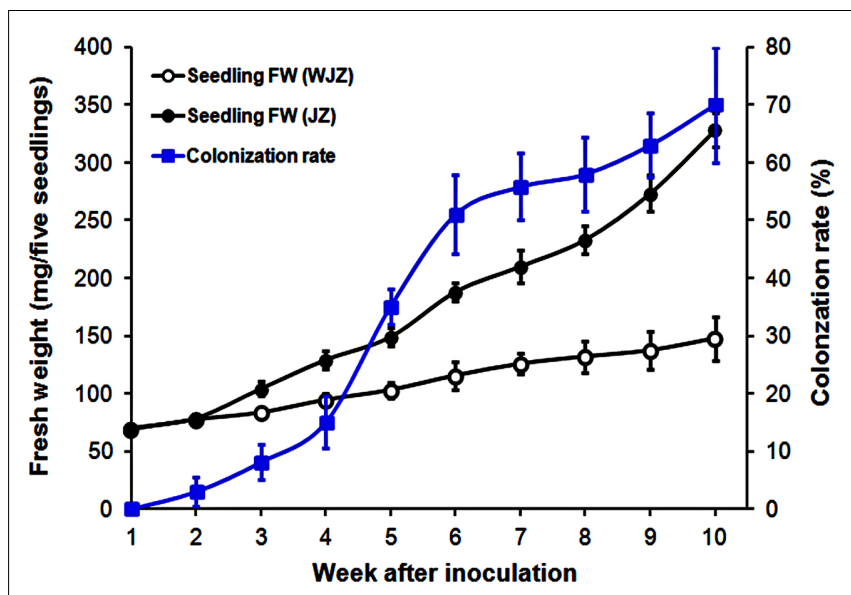

FIGURE 1 | Fresh weight (mg) of five Rhododendron fortunei seedlings inoculated (JZ) and uninoculated (WJZ) with an ericoid mycorrhizal (ERM) fungus (Oidiodendron maius var. maius Om19) as well as colonization rate of seedlings by the fungus during a 10-week growth period. Bars represent standard errors $(n=6)$. cells in week 3 and became clearly present in week 4 . Root epidermal and cortical cells completely filled with mycelium in week 6 (Figure 2A). There was no mycelium on any root surface of control (WJZ) seedlings (Figure 2B). SEM observation also showed that mycelium heavily surrounded roots of JZ seedlings (Figure 2C) but the surfaces of control roots were clear (Figure 2D).

The second experiment, designed to study $\mathrm{N}$ uptake and biomass accumulation, showed that Om19-colonized seedlings (JZ) were much larger with more roots than those of control (WJZ) during a 6-week growth period (Figure 3). Total dry weight (root and shoot) of $50 \mathrm{JZ}$ seedlings was $274 \mathrm{mg}$ compared to $187 \mathrm{mg}$ of $50 \mathrm{WJZ}$ seedlings (Figure 4), a $46.6 \%$ increase. Total $\mathrm{N}$ in $50 \mathrm{JZ}$ seedlings was $4.48 \mathrm{mg}$ compared to $3.28 \mathrm{mg}$ of $50 \mathrm{WJZ}$ seedlings, a $36.6 \%$ increase.

\section{Transcriptome Sequencing Output and Assembly}

After stringent quality checking and data cleaning, the Illumina sequencing platform generated 51.7 million, 90-bp long reads comprising $4.66 \mathrm{~Gb}$ nucleotides from $\mathrm{WJZ}$ and 50.6 million reads consisting of $4.56 \mathrm{~Gb}$ nucleotides from JZ (Table 1). The $\mathrm{N}$ percentage was $0.01 \%$ for both $\mathrm{JZ}$ and WJZ, suggesting that the proportion of unknown nucleotides in reads were extremely low. The sequencing quality was high since Q20 scores for reads of JZ and WJZ were 97.30 and $97.27 \%$, respectively. The Q scores are defined as a property that is logarithmically related to the base calling error probabilities, and the Q20 means 1 error per 100 sequenced bases (Ewing et al., 1998). The reads were de novo assembled using the Trinity into 115,917 contigs with a N50 of $428 \mathrm{bp}$ ( $50 \%$ of the assembled contigs had 428 bp or longer) for WJZ and 151,974 contigs with a N50 of 346 bp for JZ. The contigs were further assembled into scaffolds with paired-end read joining and gap-filling. Scaffolds were then assembled into 68,627 unigenes with a mean length of 458 bp for WJZ and 87,692 unigenes with a mean length of $410 \mathrm{bp}$ for JZ (Table 1). Using the same strategy, a total of 70,720 unigenes with a mean length of 570 bp were obtained from both WJZ and JZ unigenes, which were called as all unigenes (Table 1).

\section{Functional Annotation of all Unigenes}

For validation and annotation of the assembled unigenes, all 70,720 unigenes were searched against six databases using Basic Local Alignment Search Tool (BLASTX) with an $E$-value $<10^{-5}$, which resulted in 45,058, 36,399, 28,048, 24,986, 15,105, and 35,283 unigenes that were annotated to Nr, NT, Swiss-Prot, KEGG, COG, and GO databases, respectively. As a result, a total of $46,959(66.4 \%)$ all unigenes were annotated to one or more of the databases. The $E$-value distribution of the top hits in the $\mathrm{Nr}$ database revealed that $45 \%$ of the mapped sequences showed significant homology (less than $1.0 E$ - 45) (Supplementary Figure S1A), and 69.3 and $28.2 \%$ of the sequences had similarities greater than 60 and 80\%, respectively (Supplementary Figure S1B). Of which $49.3 \%$ of the unigenes were homologous with sequences of Vitis vinifera; $12.7,12.2$, and $6.4 \%$ of the unigenes had a significant similarity with the sequences of Ricinus 

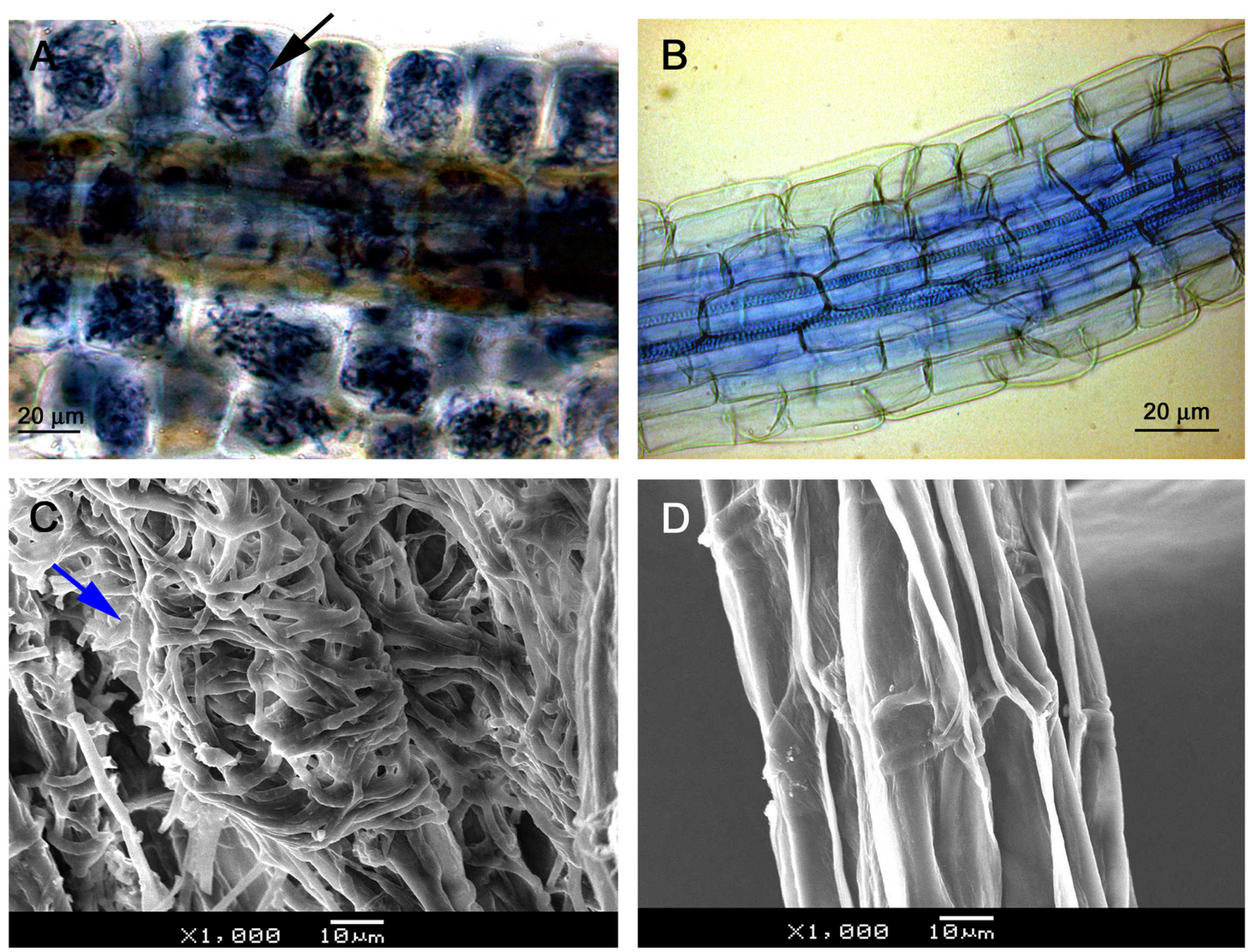

FIGURE 2 | Microscopic observation of Rhododendron fortunei roots infected by an ERM fungus (Oidiodendron maius var. maius strain Om19). Root epidermal and cortical cells were completely filled with mycelium in week 6 as indicated by arrow (A); no mycelium on root surface of seedlings (B); SEM observation showing mycelia heavily surrounded roots (arrow) of seedlings inoculated with the fungus (C); and the surface of control roots was clear (D).

communis, Populus trichocarpa, and Glycine max, respectively (Supplementary Figure S1C).

The GO database comprises three ontologies: molecular function, cellular components, and biological processes. The basic units of GO are the "GO terms," each belongs to a type of ontology. A total of 35,283 unigenes were assigned to 57 GO terms consisting of three domains: biological process, cellular component, and molecular function (Supplementary Figure S2). Some of the most common processes included cellular process, cell, cell part, and organelle. In contrast, only four genes were assigned to metallochaperone activity, and one gene was assigned to virion.

The COG database contains classifications of orthologous gene products. The 15,105 unigenes which were annotated to COG database were distributed to 25 COG categories (Figure 5). Among the 25 COG categories, general function prediction represented the largest group (4,891 unigenes, accounting for $32.38 \%$ ), followed by transcription $(3,351,22.18 \%)$, and posttranslational modification/protein turnover/chaperones (2685, $17.78 \%)$. The smallest groups were nuclear structure $(4,0.03 \%)$ and extracellular structures $(5,0.03 \%)$.
To identify the biological pathways, 70,720 all unigenes were mapped to KEGG pathways, of which 24,986 were annotated to KEGG, and assigned to the 128 KEGG pathways. The most highly represented categories were metabolism pathway (6,241 unigenes), biosynthesis of secondary metabolites $(2,486$ unigenes), and endocytosis pathway (1,565 unigenes). However, only six unigenes were assigned to biotin metabolism pathway and four to betalain biosynthesis pathway. Additionally, a number of unigenes were only annotated to a single pathway.

\section{Functional Annotation of DEGs}

A total of 48,148 unigenes were upregulated and 22,541 were downregulated due to the Om19 colonization using the Cufflinks (Trapnell et al., 2010). According to the threshold of the false discovery rate (FDR $\leq 0.001)$ and the absolute value of $\log 2$ Ratio $(\geq 1), 16,892$ unigenes were found to be significantly differentially expressed. Of which 14,364 were upregulated and 2,528 were downregulated. The number of upregulated genes was over fivefold more than that of downregulated genes. A scatter plot showed a positive relationship of unigenes between JZ and WJZ (Figure 6), suggesting that the expression of most of the genes 


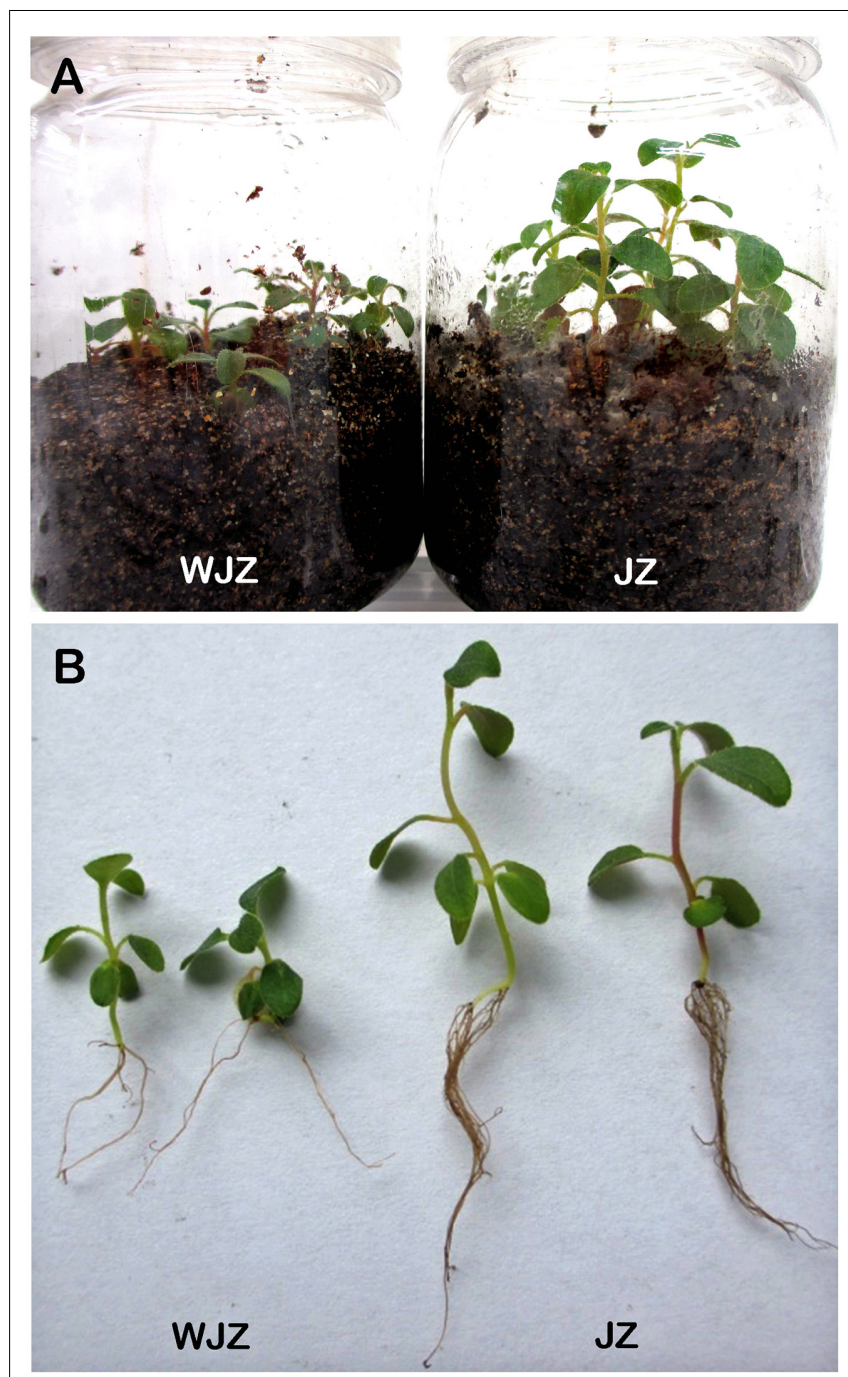

FIGURE 3 | Seedling of Rhododendron fortunei grown in a peat-based substrate inoculated (JZ) and uninoculated (WJZ) with a mycorrhizal fungus (Oidiodendron maius var. maius strain Om19) for 6 weeks (A) and representative seedlings after washing away substrate (B).

had a similar pattern and that a sizeable portion of the genes were differentially expressed due to the Om19 colonization.

Based on GO analysis, 5,939 of DEGs were further classified. They were categorized into 51 GO terms consisting of three domains: biological processes (24 terms), cellular components (15 terms), and molecular function (12 terms) (Figure 7). Within the biological processes catgory, cellular process, metabolic process, and response to stimulus were highly represented. In the cellular component category, cell and cell part dominated. In the molecular function group, observed binding and catalytic activity prevailed.

Kyoto Encyclopedia of Genes and Genomes is a large publicly available database for identifying enriched genes that may be part of metabolic or signal transduction pathways (Kanehisa et al., 2008). To explore the biological function of those differentially expressed genes, 4,620 DEGs were mapped

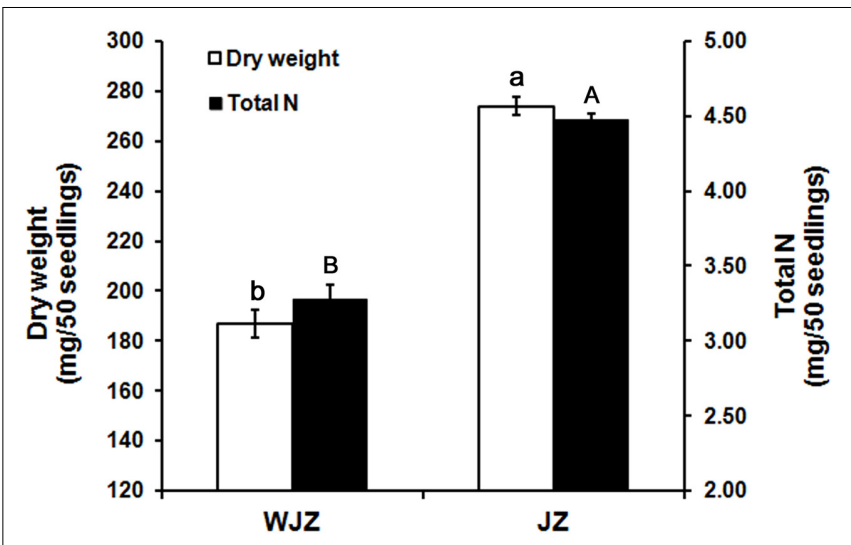

FIGURE 4 | Dry weight (mg) of 50 Rhododendron fortunei seedlings 6 weeks after inoculation (JZ) and uninoculation (WJZ) with an ERM fungus (Oidiodendron maius var. maius strain Om19) and total $N$ in the 50 seedlings, respectively, where $a$ and $b$ indicate significant dry weight differences and $\mathrm{A}$ and $\mathrm{B}$ indicate significant total $\mathrm{N}$ differences between $\mathbf{J Z}$ and $\mathbf{W J Z}$ at $\boldsymbol{P}<\mathbf{0 . 0 1}$ level based on Fisher's protected least significant difference. Bars represent standard errors $(n=6)$.

to 267 pathways in the KEGG database. These genes were enriched in several important pathways, including metabolic pathways $(1,398,30.37 \%)$, biosynthesis of secondary metabolites (696, 13.4\%), microbial metabolism in diverse environments (244, 5.29\%), plant and pathogen interaction (237, 5.14\%), plant hormone signal transduction $(233,4.83 \%)$, and starch and sucrose metabolism (193, 4.18\%).

A series of unigenes related to symbiotic processes were upregulated in Om19-colonized roots. For example, unigene38932, a homology to a gene for 1-deoxy-D-xylulose 5-phopate synthase (DXS, EC2.2.1.7) and a key enzyme in methyl-D-erythrito 4-phosphate (MEP) pathway, was upregulated by a log-fold change of 4.0. More than 10 unigenes encoding to ATP binding cassette $(\mathrm{ABC})$ transporters were upregulated by log-fold changes from 3 to 13 . Unigene29489, a homologous of GRAS-type transcription factors in legumes was also upregulated in a log-fold change of 3.4. A total of 13 unigenes homologous to early nodulin proteins (ENOD) were identified and 7 of them were upregulated by log-fold

TABLE 1 | Summary of the sequence assembly after Illumina sequencing of Rhododendron fortunei roots uninoculated (WJZ) and inoculated (JZ) with an ericoid mycorrhizal fungus (Oidiodendron maius var. Maius Om19).

\begin{tabular}{lccc}
\hline & WJZ & JZ & WJZ \& JZ \\
\hline Total clean nucleotides (bp) & $4,657,028,220$ & $4,557,459,600$ & \\
Total clean reads & $51,744,758$ & $50,638,440$ & \\
GC percentage & $50.43 \%$ & $49.47 \%$ & \\
Total number of contigs & 115,917 & 151,974 & \\
Mean length of contigs (bp) & 291 & 265 & \\
Total number of unigenes & 68,627 & 87,692 & 70,720 \\
Mean length of unigenes (bp) & 458 & 410 & 570
\end{tabular}



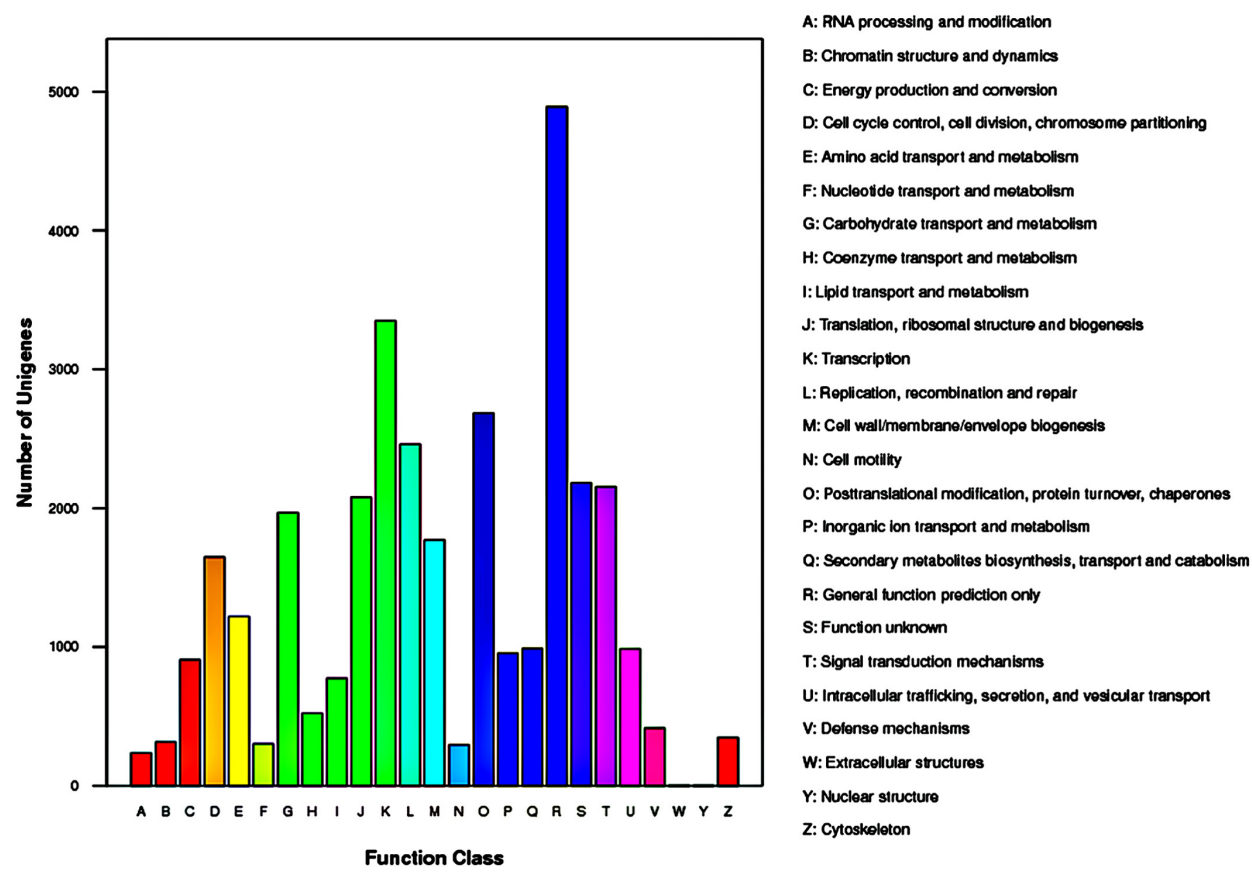

FIGURE 5 | Histogram representation of clusters of orthologous groups (COG) classification of Rhododendron fortunei transcriptome where all unigenes were assigned to 25 categories in the COG classification.

changes greater than 10. Unigene1085, the common SYM genes DMI1, DMI2, and DMI3 of Medicago truncatula (Genre et al., 2005) were also upregulated by log-fold change more than 2.6. Unigene24249, a homologous to lysine-motif (LysM) receptor kinase was upregulated by a log-fold change of 1.7. A group of unigenes related to CCaMK were upregulated by log-fold changes up to 13.7. Unigene37601, Nod factor binding lectin-nucleotide phosphohydrolase and unigene22160_All, NORK were upregulated by log-fold changes of 11.8 and 2.1, respectively. A total of 11 unigenes related to nuclear pore complex proteins (NUP) were also upregulated by log-fold changes from 11.5 to 15.6. Additionally, 251 DEGs were annotated as plant-pathogen interaction, of which 196 were upregulated by log-fold changes up to 15.2 .

Unigenes involved in nitrogen metabolism were upregulated by log-fold changes from 1 to 16 . Unigenes homologous to NRT were upregulated by log-fold changes from 1 to 13.5. Unigenes homologous to AMT family were also upregulated by log-fold changes from 1.2 to 13.9 (Supplementary Table S3), nitrate reductase and nitrite reductase upregulated by long-fold change of 2.2 and 11.4, respectively. GS and GOGAT were upregulated by log-fold change of 3 and 16.1, respectively.

The present study also identified a number of genes involving endocytosis (411, 8.91\%), Fc-gamma R-mediated phagocytosis (395, 8.56\%), glycerophospholipid metabolism (390, 8.45\%), Gonadotropin-releasing hormone $(\mathrm{GnRH})$ signal pathway (379, $8.22 \%$ ), and ether lipid metabolism (365, 7.91\%). Among them, 287 unigenes homologous to phospholipase D (PLD) (E.C. 3.1.4.4) were upregulated, of which 87 were up by longfold changes greater than 10. PLD occurs in endocytosis,

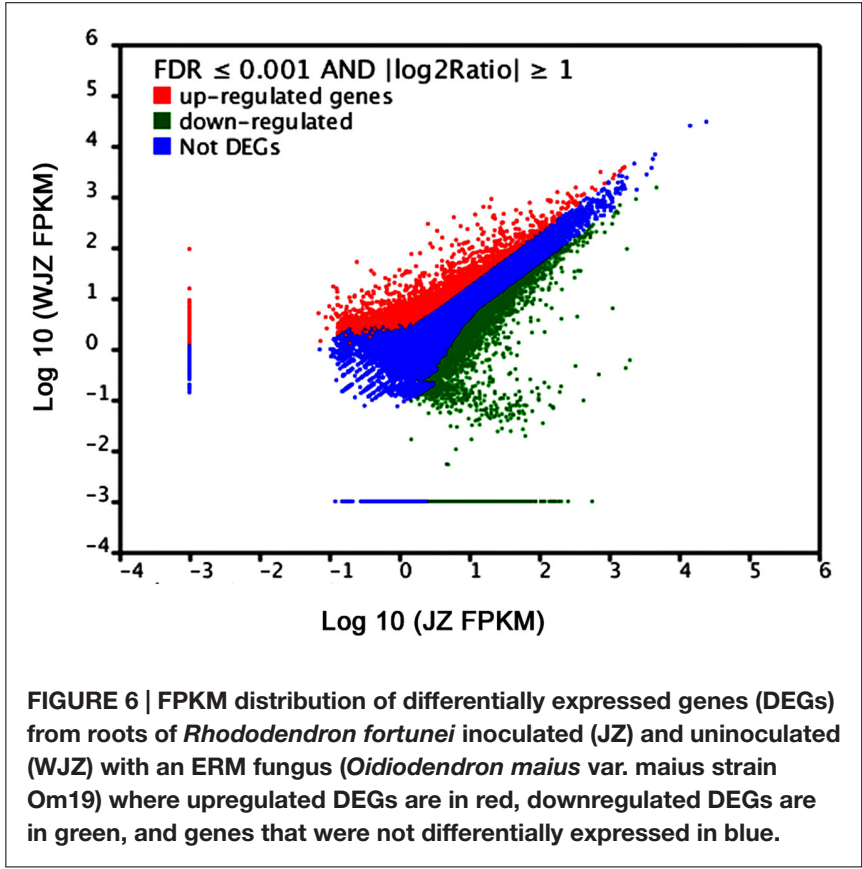

ether lipid metabolism, Fc-gamma R-mediated phagocytosis, glycerophospholipid metabolism, and GnRH signal pathways. Nine unigenes were homologous to extracellular-signal-regulated kinases (ERK1/2), and 7 of them were upregulated by more than 10-fold. ERK1/2 occurs in both Fc-gamma R-mediated phagocytosis and GnRH signal pathways. 
Wei et al.

Mycorrhiza-Induced Gene Expression in Rhododendron

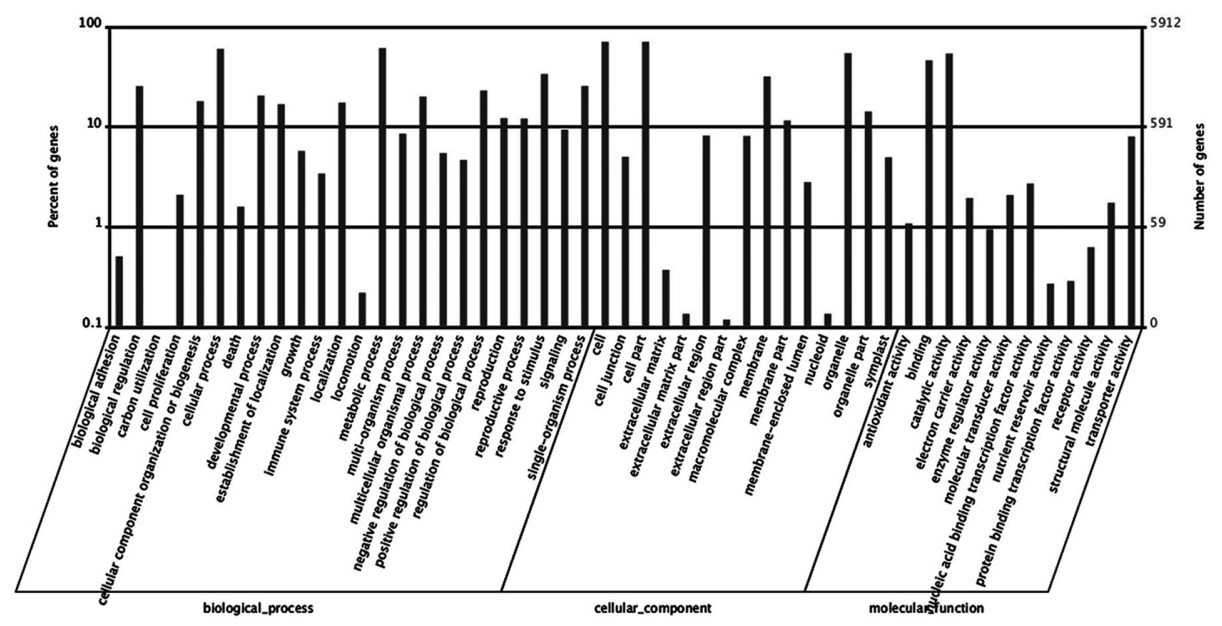

FIGURE 7 | Histogram of differentially expressed genes (LEGs) in roots of Rhododendron fortune inoculated (JZ) and uninoculated (WJZ) with an ERM fungus (Oidiodendron mains var. mains strain Om19) which were classified based on gene ontology (GO).

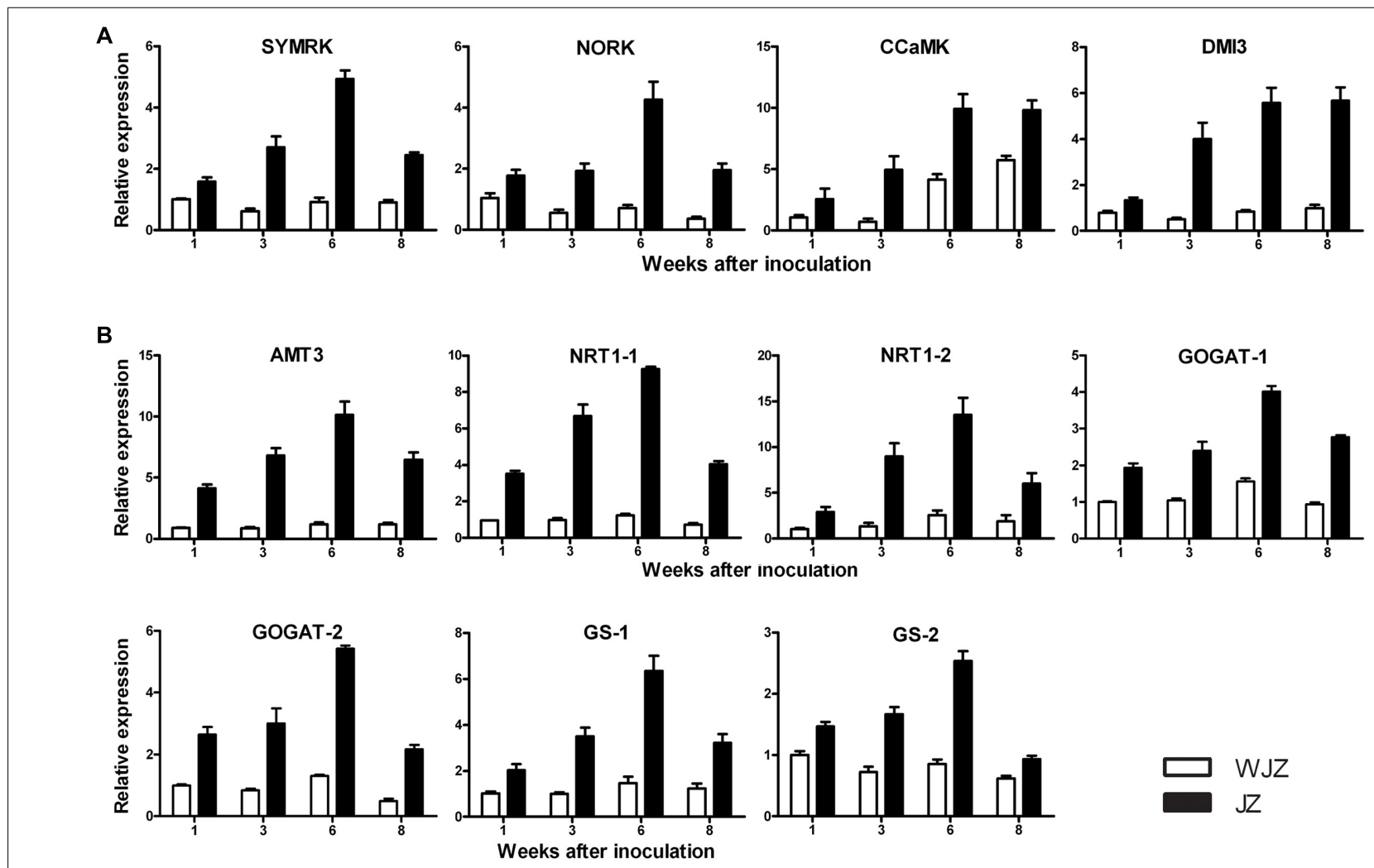

FIGURE 8 | qRT-PCR analysis of 11 selected differentially expressed genes in roots of Rhododendron fortune inoculated (JZ) and uninoculated (WJZ) with an ERM fungus (Oidiodendron mains var. mains strain Om19). The relative expression levels were normalized and calibrated according to the $2^{-} \Delta \Delta \mathrm{CT}$ method. For a given gene, the relative expression level was expressed as mean $\pm S E(n=3)$. (A) Genes related to symbiosis (SymRK, NORK, CCaMK, and DM1), (B) Genes related to N uptake and metabolism (AMT3, NRT-1-1, NRT1-2, GOGAT-1, GOGAT-2, GS-1, and GS-2).

qRT-PCR analysis showed that the selected 11 DEG were highly unregulated in JZ roots ranging from 1.5 to 9.5 fold compared to their expressions in WJZ roots (Figure 8). The relative expression of BEGs homologous to SymRK, NORK,
CCAMK, and DMI3 continuously increased from weeks 1 to 6 in JZ roots; SymRK and NORK decreased in week 8; and $C C a M K$, and $D M I 3$ reached a plateau on week 8 (Figure 8A). The expression of these genes in WJZ roots varied to a limited

Frontiers in Plant Science | www.frontiersin.org

9

October 2016 | Volume 7 | Article 1594 
extent over the 8-week period except for $C \mathrm{CaMK}$ that increased from week 6 to 8. DEGs homologous to N uptake (AMT3, NRT11, and NRT1-2) also continuously increased from weeks 1 to 6 , and decreased in week 8 (Figure 8B). Correspondingly, GOGAT1, GOGAT-2, GS-1, and GS-2 showed the same expression pattern as AMT3, NRT1-1, and NRT1-2. Whereas the expression AMT3, NRT1-1, and NRT1-2 as well as GOGAT-1, GOGAT-2, and GS-1 in WJZ roots slightly increased.

\section{DISCUSSION}

The present study devised a simple system for establishing the symbiotic relationship between $R$. fortunei seedlings and an ERM fungus (O. maius). Om19 infection and colonization can be examined, and plant growth and $\mathrm{N}$ uptake can be quantified. Based on the system, high-quality RNA can be extracted from plant samples. Using the Illumina HiSeq ${ }^{\mathrm{TM}} 2000$, we were able to obtain high-quality transcript sequences as indicated by low proportion of undefined nucleotides (Ns) and low base-calling error probability. Our study is the first to document such a large number of genes involved in the symbiosis between an ERM fungus and $R$. fortunei and the genetic underpinning of mycorrhizal-mediated increase in $\mathrm{N}$ uptake and host plant growth.

\section{Om19 Colonization and Plant Growth}

Since the peat-based substrate was autoclaved, and inoculation was performed under sterile conditions, the inoculated Om19 should be the only microbe in the system. The observed microbial infection and plant growth differences should be solely attributed to the interaction between the Om19 and R. fortunei. The colonization data suggested that by week $6, R$. fortunei roots were thoroughly infected by Om19 (Figure 1). Om19 colonization enhanced root growth as greater root numbers and longer root lengths were observed in seedlings colonized by Om19 than the control (Figure 3). Large root systems result in a large root surface and shorter average half distance between root axes in the substrate for effective capture of nutrient elements (Chen and Gabelman, 2000). As a result, total $\mathrm{N}$ absorbed by Om19-colonized plants was higher than the control plants (Figure 4). Increased $\mathrm{N}$ uptake promoted plant growth, thus the dry weight of seedlings inoculated with Om19 was significantly greater than the control seedlings (Figure 4).

\section{Genes Related to Symbiosis}

RNA-Seq identified 16,892 genes that were significantly differentially expressed in Om19-colonized roots. The highly expressed genes such as DXS in MEP pathway could result in the production of strigolactones. Strigolactones may be able to induce hyphal growth and branching of Om19. ABC transporters, particularly G-subfamily ones are thought to be putative strigolactone export component in Petunia (Kretzschmar et al., 2012). Unigenes homologous to ABC transporters were highly expressed in colonized roots of R. fortunei. GRAS-type transcription factors which were implicated in the regulation of strigolactone biosynthesis in legumes (Gutjahr and Parniske, 2013) were upregulated in $R$. fortunei. Subsequently, ENOD and the common SYM genes DMI1, DMI2, and DMI3 were found to be significantly upregulated. qRT-PCR analysis also showed that SYMRK, NORK, $C C a M K$, and DMI3 increasingly expressed from weeks 1 to 6 (Figure 8A). These genes are required for PPA induction in Medicago truncatula (Genre et al., 2005), and DMI3 is required for a subset of genes to be induced during PPA formation (Siciliano et al., 2007). A gene homologous to LysM receptor kinase which is involved in symbiotic signal perception at the root plasma membrane (Broghammer et al., 2012) was increasingly expressed. These results suggest that the interaction between Om19 and $R$. fortunei probably occurred in weeks 1 to 3 , and the symbiosis was probably well established before week 6 . The significantly increased expression of CCaMKs, DMI1, DMI2, and DMI3, NORK, and NUP during the early stage of Om19 colonization of $R$. fortunei may suggest that the ERM fungus Om19 has similar processes as AMs in symbiosis with its host plants.

This study also identified a number of genes involving endocytosis, Fc-gamma R-mediated phagocytosis, glycerophospholipid metabolism, GnRH signal pathway, and ether lipid metabolism, which were significantly differentially expressed during the symbiosis. Endocytosis, a new and exciting research area in plant biology (Robinson, 2015), could be an important process for plants interacting with ERM fungi. It might be possible that PPA formation is associated with endocytosis. Phagocytosis plays an essential role in host-defense mechanisms through the uptake and destruction of infectious pathogens in animals. After opsonization with antibodies (IgG), foreign extracellular materials are recognized by Fc gamma receptors. Cross-linking of $\mathrm{Fc}$ gamma receptors initiates a variety of signals mediated by tyrosine phosphorylation of multiple proteins, which lead through the actin cytoskeleton rearrangements and membrane remodeling to the formation of phagosomes (Indik et al., 1995). Phosphoglycerolipids are essential structural constituents of membranes and some also have important cell signaling roles (Janda et al., 2013). One of the most important signaling lipids in plants is phosphatidic acid (Zhang and Xiao, 2015), which can activate or inactivate protein kinases and/or protein phosphatases involved in hormone signaling. It can also activate NADPH oxidase leading to the production of reactive oxygen species. GnRH was identified in animals, whose secretion from the hypothalamus acts upon its receptor in the anterior pituitary to regulate the production and release of the gonadotropins, luteinizing hormone (LH), and follicle-stimulating hormone (FSH). The $\mathrm{GnRH}$ is coupled to $\mathrm{Gq} / 11$ proteins to activate phospholipase C which transmits its signal to diacylglycerol (DAG) and inositol 1, 4, 5-trisphosphate (IP3). DAG activates the intracellular protein kinase C (PKC) pathway and IP3 stimulates release of intracellular calcium (Conn, 1994). To the best of our knowledge, there has been no report about the involvement of these genes in mycorrhizal colonization. Confirming their existence and their roles in the symbiotic relationships requires further investigation. 
The Om19 colonization also induced plant defense responses as 251 DEGs were annotated to plant-pathogen interactions. Since seedlings colonized by Om19 were healthy and larger than the control ones, the defense reactions induced by Om19 could be mild and temporary, which is similar to the colonization of AM fungi with their hosts (Parniske, 2008). The defense responses may also have little effect on mycorrhizal infection (Hause and Fester, 2005; El-Khallal, 2007) as colonization rates increased (Figure 1). However, exact mechanisms underlying the host plant defense responses to Om19 infection and whether the responses affects its infection is currently unclear.

\section{N Metabolism}

Inorganic $\mathrm{N}$ in the soil is preferentially taken up by AM fungi as $\mathrm{NO}_{3}{ }^{-}$or ammonium. Once the $\mathrm{N}$ is absorbed by the extraradical mycelia, it is converted into arginine for transport into intra-radical mycelia (Gomez et al., 2009; Tian et al., 2010). The arginine is broken down through the urease cycle into ammonium for transport into the plant (Govindarajulu et al., 2005). The present study used $\mathrm{NO}_{3}{ }^{-}$as $\mathrm{N}$ source. The total $\mathrm{N}$ was substantially higher in Om19-colonized seedlings (Figure 4). RNA-Seq analysis identified 11 DEGs that were homologous to AMTs, and their expressions in Om19 colonized roots were upregulated by log-fold-changes from 1.3 to 13.9 compared to the uninoculated control (Supplementary Table S3). qRTPCR analysis of AMT3 also showed that its expression highly increased in Om19-colonized roots (Figure 8B). These results may suggest that mycelia of Om19, similar to AM fungi, absorbed $\mathrm{NO}_{3}{ }^{-}$, converted it into arginine, and then released ammonium to plants. Increased ammonium in plants may trigger GS and GOGAT activities. This study identified three unigenes homologous to GS, and nine unigenes to GOGAT. qRT-PCR analysis showed that the expression of GS-1 and GS-2 almost linearly increased in Om19 colonized roots from weeks 1 to 6 after inoculation (Figure 8B). GS and GOGAT are key metabolic enzymes that synthesize glutamine and glutamate, leading to the entrance of organic nitrogen in cellular metabolic pathways such as the biosynthesis of amino acids, nucleic acids and complex polysaccharides.

In addition to the aforementioned fungi mediated $\mathrm{N}$ uptake, Om19-colonized roots could also directly absorb $\mathrm{NO}_{3}{ }^{-}$since unigenes homologous to NRTs were significantly upregulated. NTRs have two families: NRT1 and NRT2. Members of the NRT1 family mainly regulate the LATS and members of the NRT2 family regulate HATS only (Kumar et al., 2003). We identified eight upregulated DEGs homologous to the NRT1 family. qRT-PCR analysis showed that NRT-1-1 and NRT1-2 were highly upregulated in Om19-colonized roots (Figure 8B). Recent studies showed that NRT1.1 from Arabidopsis is actually a dual-affinity transporter regulating $\mathrm{NO}_{3}{ }^{-}$uptake by changing its affinity for $\mathrm{NO}_{3}{ }^{-}$depending on the availability of $\mathrm{NO}_{3}{ }^{-}$in the soil (Tsay, 2014; Sun and Zheng, 2015). It is unknown at present if NRT1-1 or NRT1-2 in R. fortunei plays the same roles as NRT1.1 in Arabidopsis. The increased expression of NRT1-1 and NRT1-2 does suggest that NRTs were active in Om19-colonized roots.

The present study demonstrates that $R$. fortunei can use $\mathrm{NO}_{3}{ }^{-}$ as an $\mathrm{N}$ source under acidic growing conditions. Seedlings grew healthy in the peat-based substrate with a $\mathrm{pH}$ of 5.2, and seedling biomass linearly increased over time (Figure 1). In addition to using inorganic N, ERM fungi are capable of enzymatically degrading organic N from substrates (Read, 1996; Yang and Goulart, 1997). The increased expression of AMT3, NRT1-1, and NRT1-2 from weeks 1 to 6 may suggest that the colonized roots mainly absorbed available $\mathrm{NO}_{3}{ }^{-}$during this growth period. Since no additional nutrients were provided after transplanting, the decreased expression of these genes in week 8 makes us speculate that Om19 might start to degrade the peat substrate, and seedlings might begin to take up organic N. Recent study showed that $O$. maius symbionent expressed a full complement of plant cell wall-degrading enzymes in symbiosis, suggesting its saprotrophic ability in sphagnum peat (Kohler et al., 2015). At this point, whether Om19 enzymatically degraded organic $\mathrm{N}$ from the peat requires further investigation.

Nevertheless, the total $\mathrm{N}$ in 50 seedlings colonized by Om19 was $36.59 \%$ greater than the control seedlings (Figure 4), suggesting that Om19-colonization contributed significantly to $\mathrm{N}$ absorption. The increased uptake is likely attributed to Om19-mediated bioavailability of $\mathrm{N}$ and direct $\mathrm{NO}_{3}{ }^{-}$ absorption. A total of 51 DEGs were identified which are related to the nitrogen metabolism, and most of the DEGs were dramatically upregulated in Om19 colonized roots. Due to the increased $\mathrm{N}$ uptake, many pathways including energy and nutrient metabolism, glycolysis/gluconeogenesis, pentose phosphate pathway, TCA cycle, plant hormone signal transduction, starch and sucrose metabolism, and amino sugar and nucleotide sugar metabolism became more active as most of the genes mapping to the above pathways were greatly upregulated in roots inoculated with Om19. The Om19colonized seedlings are metabolically more active than the control seedlings. Thus, Om19-colonized seedlings grew significantly larger than control ones.

\section{CONCLUSION}

Ericaceous shrubs such as Calluna vulgaris, Rhododendron spp., and Vaccinium spp. occur both in open heathland communities and in forest ecosystems as understory vegetation (Read, 1996). Soils in those habitats are typically low in available nutrients and plants grown in such soils are often stressed by different factors such as low $\mathrm{pH}$, metal availability, water availability, and high or low temperatures (Read, 1996; Cairney and Meharg, 2003). Cortical cells of ericaceous plants never form root hairs as those in the other plant families. Thus, the ability to form symbiotic relationships with ERM fungi is consider to be critical to the success of ericaceous plants in such stressful soil conditions (Read, 1996; Cairney and Meharg, 2003). The present study documented that 16,892 genes were differentially expressed in an ERM fungus colonized roots. Such a large number of gene expressions may suggest that the long-history co-evolution between $R$. fortunei and ERM fungi has fine-tuned plant genetic networks that particularly fit the unique ecosystem where soils are poor and acidic in a mutually beneficial way. 


\section{AUTHOR CONTRIBUTIONS}

DP and CZ conceived and designed the experiments. XW conducted the experiments, analyzed the data, and drafted the manuscript. JC participated in data analysis, wrote, and revised the manuscript. The final version was approved by all authors.

\section{FUNDING}

The authors would like to thank National Natural Science Foundation of China (No. 30972409) and the Scientific Research Foundation of Graduate School at the Fujian Agriculture and Forestry University (324-1122YB026) for supporting this study.

\section{REFERENCES}

Akiyama, K., Matsuzaki, K.-I., and Hayashi, H. (2005). Plant sesquiterpenes induce hyphal branching in arbuscular mycorrhizal fungi. Nature 435, 824-827. doi: 10.1038 /nature 03608

Ané, J. M., Kiss, G. B., Riely, B. K., Penmetsa, R. C., Oldroyd, G. E., Ayax, C., et al. (2004). Medicago truncatula DMI1 required for bacterial and fungal symbioses in legumes. Science 303, 1364-1367. doi: 10.1126/science. 1092986

Ashburner, M., Ball, C. A., Blake, J. A., Botstein, D., Butler, H., Cherry, J. M., et al. (2000). Gene ontology: tool for the unification of biology. Nat. Genet. 25, 25-29. doi: $10.1038 / 75556$

Audic, S., and Claverie, J.-M. (1997). The significance of digital gene expression profiles. Genome Res. 7, 986-995.

Benjamini, Y., and Yekutieli, D. (2001). The control of the false discovery rate in multiple testing under dependency. Ann. Stat. 29, 1165-1188.

Biermann, B., and Linderman, R. G. (1981). Quantifying vesicular-arbuscular mycorrhizae: a proposed method towards standardization. New Phytol. 87, 63-67. doi: 10.1111/j.1469-8137.1981.tb01690.x

Bonfante, P., and Genre, A. (2010). Mechanisms underlying beneficial plantfungus interactions in mycorrhizal symbiosis. Nat. Commun. 1:48. doi: 10.1038/ncomms 1046

Bonfante-Fasolo, P., and Gianinazzi-Pearson, V. (1979). Ultrastructural aspects of endomycorrhiza in the Ericaceae. New Phytol. 83, 739-744. doi: 10.1111/j.14698137.1979.tb02304.x

Broghammer, A., Krusell, L., Blaise, M., Sauer, J., Sullivan, J. T., Maolanon, N., et al. (2012). Legume receptors perceive the rhizobial lipochitin oligosaccharide signal molecules by direct binding. Proc. Natl. Acad. Sci. U.S.A. 109, 13859-13864. doi: 10.1073/pnas.1205171109

Bucking, H., and Kafle, A. (2015). Role of arbuscular mycorrhizal fungi in the nitrogen uptake of plants: current knowledge and research gaps. Agronomy 5, 587-612. doi: 10.3390/agronomy5040587

Cairney, J. W. G., and Meharg, A. A. (2003). Ericoid mycorrhiza: a partnership that exploits harsh edaphic conditions. Euro. J. Soil Sci. 54, 735-740. doi: 10.1046/j.1351-0754.2003.0555.x

Chen, J., and Gabelman, W. H. (2000). Morphological and physiological characteristics of tomato roots associated with potassium-acqusisiton efficiency. Sci. Hort. 83, 213-225. doi: 10.1016/S0304-4238(99) 00079-5

Conesa, A., Gotz, S., Garcia-Gomez, J. M., Terol, J., Talón, M., and Robles, M. (2005). Blast2GO: a universal tool for annotation, visualization and analysis in functional genomics research. Bioinformatics 21, 3674-3676. doi: 10.1093/bioinformatics/bti610

Conn, P. M. (1994). Gonadotropin-releasing hormone and its analogs. Ann. Rev. Med. 45, 391-405. doi: 10.1146/annurev.med.45.1.391

Economou, A., and Read, P. (1984). In vitro shoot proliferation of Minnesota deciduous azaleas. HortScience 19, 60-61.

El-Khallal, S. M. (2007). Induction and modulation of resistance in tomato plants against Fusarium wilt disease by bioagent fungi (arbuscular mycorrhiza)

\section{ACKNOWLEDGMENTS}

Dr. Dongfang $\mathrm{Wu}$ at Ecloudbio Co. and Hao Sun at the Institute of Applied Ecology, Chinese Academy of Science, Shenyang, China for their assistance in transcriptome data analysis, and Mrs. Barb Henny for critical review of the manuscript.

\section{SUPPLEMENTARY MATERIAL}

The Supplementary Material for this article can be found online at: http://journal.frontiersin.org/article/10.3389/fpls.2016. 01594

and/or hormonal elicitors (jasmonic acid and salicylic acid): 2-changes in the antioxidant enzymes, phenolic compounds and pathogen related-proteins. Aust. J. Basic Appl. Sci. 1, 717-732.

Endre, G., Kereszt, A., Kevei, Z., Mihacea, S., Kalo, P., and Kiss, G. B. (2002). A receptor kinase gene regulating symbiotic nodule development. Nature 417, 962-966. doi: 10.1038/nature00842

Ewing, B., Hillier, L., Wendl, M. C., and Green, P. (1998). Base-calling of automated sequencer traces using phred. I. Accuracy assessment. Genome Res. 8, 175-185. doi: 10.1101/gr.8.3.175

Genre, A., Chabaud, M., Timmers, T., Bonfante, P., and Barker, D. G. (2005). Arbuscular mycorrhizal fungi elicit a novel intracellular apparatus in Medicago truncatula root epidermal cells before infection. Plant Cell 17, 3489-3499. doi: 10.1105/tpc.105.035410

Gomez, S. K., Javot, H., Deewatthanawong, P., Torres-Jerez, I., Tang, Y., Blancaflor, E. B., et al. (2009). Medicago truncatula and Glomus intraradices gene expression in cortical cells harboring arbuscules in the arbuscular mycorrhizal symbiosis. BMC Plant Biol. 9:10. doi: 10.1186/1471-2229-9-10

Govindarajulu, M., Pfeffer, P. E., Jin, H. R., Abubaker, J., Douds, D. D., Allen, J. W., et al. (2005). Nitrogen transfer in the arbuscular mycorrhizal symbiosis. Nature 435, 819-823. doi: 10.1038/nature03610

Grabherr, M. G., Haas, B. J., Yassour, M., Levin, J. Z., Thompson, D. A., Amit, I., et al. (2011). Full-length transcriptome assembly from RNA-Seq data without a reference genome. Nat. Biotechnol. 29, 644-652. doi: 10.1038/nbt.1883

Gutjahr, C., and Parniske, M. (2013). Cell and developmental biology of arbuscular mycorrhiza symbiosis. Ann. Rev. Cell Devel. Biol. 29, 593-617. doi: 10.1146/annurev-cellbio-101512-122413

Harrison, M. J. (2005). Signaling in the arbuscular mycorrhizal symbiosis. Ann. Rev. Microbiol. 59, 19-42. doi: 10.1146/annurev.micro.58.030603.123749

Hause, B., and Fester, T. (2005). Molecular and cell biology of arbuscular mycorrhizal symbiosis. Planta 221, 184-196. doi: 10.1007/s00425-004-1436-x

Indik, Z. K., Park, J. G., Hunter, S., and Schreiber, A. D. (1995). The molecular dissection of Fc gamma receptor mediated phagocytosis. Blood 86, 4389-4399.

Iseli, C., Jongeneel, C. V., and Bucher, P. (1999). ESTScan: a program for detecting, evaluating, and reconstructing potential coding regions in EST sequences. ISMB 99, 138-148.

Janda, M., Planchais, S., Djafi, N., Martinec, J., Burketova, L., Valentova, O., et al. (2013). Phosphoglycerolipids are master players in plant hormone signal transduction. Plant Cell Rep. 32, 839-851. doi: 10.1007/s00299-0131399-0

Kaló, P., Gleason, C., Edwards, A., Marsh, J., Mitra, R. M., Hirsch, S., et al. (2005). Nodulation signaling in legumes requires NSP2, a member of the GRAS family of transcriptiona regulators. Science 308, 1786-1789. doi: 10.1126/science. 1110951

Kanehisa, M., Araki, M., Goto, S., Hattori, M., Hirakawa, M., Itoh, M., et al. (2008). KEGG for linking genomes to life and the environment. Nucleic Acids Res. 36(Suppl. 1), D480-D484. doi: 10.1093/nar/gkm882

Karandashov, V., and Bucher, M. (2005). Symbiotic phosphate transport in arbuscular mycorrhizas. Trends Plant Sci. 10, 22-29. doi: 10.1016/j.tplants.2004.12.003 
Knief, C. (2014). Analysis of plant microb interactions in the eaa of next generation sequencing technologies. Front. Plant Sci. 5:216. doi: 10.3389/fpls.2014.00216

Kohler, A., Kuo, A., Nagy, L. G., Morin, E., Barry, K. W., Buscot, F., et al. (2015). Convergent losses of decay mechanisms and rapid turnover of symbiosis genes in mycorrhizal mutualists. Nat. Genet. 47, 410-415. doi: 10.1038/ng.3223

Kosola, K. R., Workmaster, B. A. A., and Spada, P. A. (2007). Inoculation of cranberry (Vaccinium macrocarpon) with the ericoid mycorrhizal fungus Rhizoscyphus ericae increases nitrate influx. New Phytol. 176, 184-196. doi: 10.1111/j.1469-8137.2007.02149.x

Kretzschmar, T., Kohlen, W., Sasse, J., Borghi, L., Schlegel, M., Bachelier, J. B., et al. (2012). A petunia $A B C$ protein controls strigolactone-dependent symbiotic signalling and branching. Nature 483, 341-344. doi: 10.1038/nature10873

Kumar, A., Silim, S., Okamoto, M., Siddiqi, M. Y., and Glass, A. D. (2003). Differential expression of three members of the AMT1 gene family encoding putative high-affinity NH4+ transporters in roots of Oryza sativa subspecies indica. Plant Cell Environ. 26, 907-914. doi: 10.1046/j.1365-3040.2003.01023.x

Lévy, J., Bres, C., Geurts, R., Chalhoub, B., Kulikova, O., Duc, G., et al. (2004). A putative $\mathrm{Ca} 2+$ and calmodulin-dependent protein kinase required for bacterial and fungal symbioses. Science 303, 1361-1364. doi: 10.1126/science. 1093038

Li, R., Yu, C., Li, Y., Lam, T., Yiu, S., Kristiansen, K., et al. (2009). SOAP2: an improved ultrafast tool for short read alignment. Bioinformatics 25, 1966-1967. doi: 10.1093/bioinformatics/btp336

Martin, L. B., Fei, Z., Giovannoni, J. J., and Rose, J. K. C. (2013). Catalyzing plant science research with RNA-Seq. Front. Plant Sci. 4:66. doi: 10.3389/fpls.2013.00066

Mitra, R. M., Gleason, C. A., Edwards, A., Hadifield, J., Downier, J. A., Oldroyd, G. E., et al. (2004). A Ca2+/calmodulin-dependent protein kinase required for symbiotic nodule development: gene identification by transcript-based cloning. Proc. Natl. Acad. Sci. U.S.A. 101, 4701-4705. doi: 10.1073/pnas.0400595101

Molina, R., and Palmer, J. (1982). "Isolation, maintenance, and pure culture manipulation of ectomycorrhizal fungi," in Methods and Principles of Mycorrhizal Research, ed. N. C. Schenck (Saint Paul, MN: American Phytopathological Society), 115-129.

Mortazavi, A., Williams, B. A., McCue, K., Schaeffer, L., and Wold, B. (2008). Mapping and quantifying mammalian transcriptomes by RNA-Seq. Nat. Methods 5, 621-628. doi: 10.1038/nmeth.1226

Op den Camp, R., Streng, A., De Mita, S., Cao, Q., Polone, E., Liw, W., et al. (2011). LysM-type mycorrhizal receptor recruited for rhizobium symbiosis in nonlegume Parasponia. Science 331, 909-912. doi: 10.1126/science.1198181

Parniske, M. (2008). Arbuscular mycorrhiza: the mother of plant root endosymbioses. Nat. Rev. Microbiol. 6, 763-775. doi: 10.1038/nrmicro1987

Paul, E. A., and Clark, F. E. (1989). Soil Microbiology and Biochemistry. San Diego, CA: Academic Press. .

Phillips, J., and Hayman, D. (1970). Improved procedures for clearing roots and staining parasitic and vesicular-arbuscular mycorrhizal fungi for rapid assessment of infection. Trans. Br. Mycol. Soc. 55, 158-161. doi: 10.1016/S00071536(70)80110-3

Read, D. J. (1996). The structure and function of the ericoid mycorrhizal root. Ann. Bot. 77, 365-374. doi: 10.1006/anbo.1996.0044

Read, D. J., Leake, J. R., and Perez-Moreno, J. (2004). Mycorrhizal fungi as drivers of ecosystem processes in heathland and boreal forest biomes. Can. J. Bot. 82, 1243-1263. doi: 10.1139/b04-123

Read, D. J., and Perez-Moreno, J. (2003). Mycorrhizas and nutrient cycling in ecosystems-a journey towards relevance? New Phytol. 157, 475-492. doi: $10.1046 / j .1469-8137.2003 .00704 . x$

Robinson, D. G. (2015). Endocytosis: is there really a recycling from late endosomes? Mol. Plant 8, 1554-1556. doi: 10.1016/j.molp.2015.07.005

Rosen, C. J., Allan, D. L., and Luby, J. J. (1990). Nitrogen form and solution pH influence growth and nutrition of 2 Vaccinium clones. J. Amer. Soc. Hort. Sci. $115,83-89$.

Schmittgen, T. D., and Livak, K. J. (2008). Analyzing real-time PCR by comparative CT method. Nat. Protoc. 3, 1101-1108. doi: 10.1038/nprot.2008.73

Siciliano, V., Genre, A., Balestrini, R., Cappellazzo, G., deWitt, P. J. G. M., and Bonfante, P. (2007). Transcriptome analysis of arbuscular mycorrhizal roots during development of the prepenetration apparatus. Plant Physiol. 144, 1455-1466. doi: 10.1104/pp.107.097980
Smit, P., Raedts, J., Portyanko, V., Debelle, F., Gough, C., Bisseling, T., et al. (2005). NSP1 of the GRAS protein family is essential for rhizobial Nod factor-induced transcription. Science 308, 1789-1791. doi: 10.1126/science. 1111025

Smith, J. D. (1993). Uptake and Utilization of Nitrogen Sources by Cranberry Plants (Vaccinium Macrocarpon, Ait.). Madison, WI: University of Wisconsin.

Stracke, S., Kistner, C., Yoshida, S., Mulder, L., Sato, S., Kaneko, T., et al. (2002). A plant receptor-like kinase required for both bacterial and fungal symbiosis. Nature 417, 959-962. doi: 10.1038/nature00841

Sun, J., and Zheng, N. (2015). Molecular mechanism underlying the plant NRT1.1 dual-affinity nitrate transporter. Front. Physiol. 6:386. doi: 10.3389/fphys.2015.00386

Tian, C., Kasiborski, B., Koul, R., Lammers, P. J., Bucking, H., and ShacharHill, Y. (2010). Regulation of the nitrogen transfer pathway in the arbuscular mycorrhizal symbiosis: gene characterization and the coordination of expression with nitrogen flux. Plant Physiol. 153, 1175-1187. doi: 10.1104/pp.110.156430

Trapnell, C., Williams, B. A., Pertea, G., Mortazavi, A., Kwan, G., van Baren, M. J., et al. (2010). Transcript assembly and quantification by RNA-Seq reveals unannotated transcripts and isoform switching during cell differentiation. Nat. Biotechnol 28, 511-515. doi: 10.1038/nbt.1621

Tsay, Y. F. (2014). Plant science: how to switch affinity. Nature 507, 44-45. doi: 10.1038/nature13063

von Wittgenstein, N. J. J. B., Le, C. H., Hawkins, B. J., and Ehlting, J. (2014). Evolutionary classification of ammonium, nitrate, and peptide transporters in land plants. BMC Evol. Biol. 14:11. doi: 10.1186/1471-2148-14-11

Wang, Z., Gerstein, M., and Snyder, M. (2009). RNA-Seq: a revolutionary tool for transcriptomics. Nat. Genet. 10, 57-63. doi: 10.1038/nrg2484

Wei, X., Chen, J., Zhang, C., and Pan, D. (2016). A new Oidiodendron maius strain isolated from Rhododendron fortunei and its effects on nitrogen uptake and plant growth. Front. Microbiol. 7:1327. doi: 10.3389/fmicb.2016. 01327

Xiao, G., and Berch, S. M. (1992). The ericoid mycorrhizal fungi of Gaultheria shallon. Mycologia 84, 470-471. doi: 10.2307/3760201

Yang, W. Q., and Goulart, B. L. (1997). Aluminum and phosphorus interactions in mycorrhizal and nonmycorrhizal highbush blueberry plantlets. J. Am. Soc. Hort. Sci. 122, 24-30.

Ye, J., Fang, L., Zheng, H., Zhang, Y., Chen, J., Zhang, Z., et al. (2006). WEGO: a web tool for plotting GO annotations. Nucleic Acids Res. 34, W293-W297. doi: 10.1093/nar/gkl031

Yin, L., Zhang, C., and Yang, B. (2010). Characteristics of nitrogen absorbed by ericoid mycorrhizal fungi and impact on growth of Rhododendron fortunei. Sci. Agric. Sin. 43, 868-872.

Zhang, C., Hou, Y., and Dai, S. (2008). Observation on microstructure of mycorrhizal root of Rhododendron fortunei L. Acta Hort. Sin. 35, 1641-1646.

Zhang, C., Yin, L., and Dai, S. (2009). Diversity of root-associated fungal endophytes in Rhododendron fortunei in subtropical forests of China. Mycorrhiza 19, 417-423. doi: 10.1007/s00572-0090246-1

Zhang, Q., and Xiao, S. (2015). Lipids in salicylic acid-mediated defense in plants: focusing on the roles of phosphatidic acid and phosphatidylinositol 4-phosphate. Front. Plant Sci. 6:387. doi: 10.3389/fpls.2015.00387

Conflict of Interest Statement: The authors declare that the research was conducted in the absence of any commercial or financial relationships that could be construed as a potential conflict of interest.

The reviewer CF and handling Editor declared their shared affiliation, and the handling Editor states that the process nevertheless met the standards of a fair and objective review.

Copyright (c) 2016 Wei, Chen, Zhang and Pan. This is an open-access article distributed under the terms of the Creative Commons Attribution License (CC BY). The use, distribution or reproduction in other forums is permitted, provided the original author(s) or licensor are credited and that the original publication in this journal is cited, in accordance with accepted academic practice. No use, distribution or reproduction is permitted which does not comply with these terms. 